Bull. Soc. math. France

133 (3), 2005, p. $331-348$

\title{
KLOOSTERMAN-FOURIER INVERSION FOR SYMMETRIC MATRICES
}

\author{
BY OMER OFFEN
}

\begin{abstract}
We formulate a Kloosterman transform on the space of generalized Kloosterman integrals on symmetric matrices, and obtain an inversion formula. The formula is a step towards a fundamental lemma of the Jacquet type. At the same time it hints towards a conjectural relative trace formula identity, associated with the metaplectic correspondence.

RÉSUMÉ (Inversion de Kloosterman-Fourier pour les matrices symétriques)

Nous définissons une transformation de Kloosterman sur l'espace des intégrales de Kloosterman généralisées sur les matrices symétriques et nous obtenons une formule d'inversion. Cette formule est une étape vers un lemme fondamental de type de Jacquet. En même temps, elle indique une identité conjecturale de la formule des traces relative associée à la correspondance métaplectique.
\end{abstract}

\section{Introduction}

Let $F$ be a non-archimedean local field, $\mathcal{O}_{F}$ the ring of integers in $F$ and $\wp$ the maximal ideal of $\mathcal{O}_{F}$. Let $|\cdot|$ denote the normalized absolute value on $F$ so that for a uniformizer $\varpi$ of $F$ we have $|\varpi|^{-1}=\#\left(\mathcal{O}_{F} / \wp\right)$ is the size of the

Texte reçu le 23 avril 2003, révisé le 12 février 2004, accepté le 3 mars 2004

Omer Offen, Max-Planck-Institut für Mathematik, Vivatsgasse 7, D-53111 Bonn (Germany) E-mail : omer@mpim-bonn.mpg.de - Url:http://www.math.columbia.edu/ omer/ 2000 Mathematics Subject Classification. — 11F70, 11L05.

Key words and phrases. - Kloosterman integrals, Kloosterman transform, Jacquet's relative trace formula. 
residual field. Let $\psi$ be a non-trivial additive character of $F$. We recall the formula

$$
\int \widehat{f}(x) \psi\left(a x^{2}\right) \mathrm{d} x=|2 a|^{-\frac{1}{2}} \gamma(a, \psi) \int f(x) \psi\left(-a^{-1} x^{2}\right) \mathrm{d} x
$$

which we use to define the Weil constant $\gamma$. Here $a \in F^{\times}, f \in C_{c}^{\infty}(F)$ is a Schwartz function on $F$ and $\widehat{f}$ is the Fourier transform of $f$ defined by

$$
\widehat{f}(x)=\int f(y) \psi(-2 x y) \mathrm{d} y .
$$

The measure $\mathrm{d} x$ is the self dual Haar measure on $F$ with respect to $\psi$. Thus, it satisfies

$$
f(0)=|2| \int \widehat{f}(x) \mathrm{d} x .
$$

Let $N=N_{n}$ be the subgroup of upper triangular unipotent matrices in $\mathrm{GL}_{n}(F)$. Define the non-degenerate character $\theta=\theta_{n}$ of $N$ by

$$
\theta(u)=\psi\left(\sum_{i=1}^{n-1} x_{i, i+1}\right)
$$

where $u=\left(x_{i, j}\right) \in N$. The Haar measure $\mathrm{d} x$ on $F$ determines a Haar measure on $N$ and a self dual Haar measure on any finite dimensional $F$-vector space. We will use the measures determined by $\mathrm{d} x$ unless otherwise specified. Denote by $M_{m \times n}(F)$ the set of all $m \times n$ matrices with entries in $F$. Let

$$
M_{n}(F)=M_{n \times n}(F)
$$

and denote by $\mathcal{S}=\mathcal{S}_{n}$ the space of symmetric matrices

$$
\mathcal{S}=\left\{X \in M_{n}(F) ;{ }^{t} X=X\right\} .
$$

We consider the action $(u, s) \mapsto{ }^{t} u s u$ of $N$ on $\mathcal{S}$.

Definition 1.1. - An element $s \in \mathcal{S}$ is called relevant if $\theta$ is trivial on the stabilizer $N_{s}$ of $s$ in $N$.

Our objects of interest are the generalized Kloosterman integrals

$$
\omega[\Phi, \psi ; s]=\int_{N_{s} \backslash N} \Phi\left({ }^{t} u s u\right) \theta\left(u^{2}\right) \mathrm{d} u
$$

for a relevant $s \in \mathcal{S}, \Phi \in C_{c}^{\infty}(\mathcal{S})$. Let

$$
S_{n}=\mathcal{S}_{n} \cap \mathrm{GL}_{n}(F) .
$$

The orbits in $S_{n}$ are fully described in [6]. To describe a set of representatives for all orbits in $S_{n}$ we view the elements of the Weyl group as permutation matrices in $\mathrm{GL}_{n}(F)$. Thus a complete set of representatives for the orbits in $S_{n}$ is the set of all $w a$, where $w$ is the longest element in the Weyl group of a

TOME $133-2005-\mathrm{N}^{\mathrm{O}} 3$ 
standard Levi subgroup $M$ of $\operatorname{GL}_{n}(F)$ and $a$ is in the center of $M$. All relevant orbits in $\mathcal{S}_{n}$ with zero determinant contain an element of the form $\left({ }^{s}{ }_{0}\right)$, where $s \in S_{n-1}$. This is proved in [3] for Hermitian matrices. The proof for symmetric matrices is identical and we omit it here. Representatives for the relevant orbits of zero determinant are therefore given as above in terms of representatives of orbits in $S_{n-1}$. When $w=1$ and $a$ is a diagonal matrix, the stabilizer of $a$ in $N_{n}$ is trivial.

In this sense the diagonal matrices are representatives of the largest orbits. In a sense explained in [3] and [2], the Kloosterman integrals for smaller orbits, i.e. with $w \neq 1$ are determined by Kloosterman integrals of the largest orbits. For this reason, our main concern in this work is the space of Kloosterman integrals, restricted to the relevant diagonal matrices. These are of the form $a=\operatorname{diag}\left(a_{1}, \ldots, a_{n}\right)$ where $a_{1}, \ldots, a_{n-1} \in F^{\times}$and $a_{n} \in F$. We will denote by $\omega\left[\Phi, \psi ; a_{1}, \ldots, a_{n}\right]$ the Kloosterman integral $\omega\left[\Phi, \psi ; \operatorname{diag}\left(a_{1}, \ldots, a_{n}\right)\right]$.

To state our main theorem it will be convenient to introduce a normalization. We introduce the normalizing factors

$$
\sigma_{n}(a)=a_{1}^{n-1} a_{2}^{n-2} \cdots a_{n-1}, \Gamma_{n}(a, \psi)=\gamma\left(a_{1}, \psi\right)^{n-1} \gamma\left(a_{2}, \psi\right)^{n-2} \cdots \gamma\left(a_{n-1}, \psi\right)
$$

The normalized Kloosterman integral is

$$
\widetilde{\omega}^{\psi}\left[\Phi, \psi ; a_{1}, \ldots, a_{n}\right]=\Gamma_{n}(-a, \psi)\left|\sigma_{n}(a)\right|^{\frac{1}{2}} \omega\left[\Phi, \psi ; a_{1}, \ldots, a_{n}\right] .
$$

The purpose of the notation $\widetilde{\omega}^{\psi}$ is to emphasize the dependence of the normalization on the character $\psi$. Let $\Omega_{\psi, n}$ be the space of functions $\omega$ on $\left(F^{\times}\right)^{n-1} \times F$ of the form

$$
\omega\left(a_{1}, \ldots, a_{n}\right)=\widetilde{\omega}^{\psi}\left[\Phi, \psi ; a_{1}, \ldots, a_{n}\right]
$$

for some $\Phi \in C_{c}^{\infty}(\mathcal{S})$. Denote by $[\cdot, \cdot]: F^{\times} \times F^{\times} \mapsto\{ \pm 1\}$ the quadratic Hilbert symbol. It is defined by the condition $[a, b]=1$ iff $a$ is representable by the quadratic form $a=x^{2}-b y^{2}$. We define the Kloosterman transform $K_{\psi, n}$ on $\Omega_{\psi, n}$ by

$$
\begin{gathered}
K_{\psi, n} \omega\left(a_{1}, \ldots, a_{n}\right)=\int \omega\left(p_{1}, \ldots, p_{n}\right) \psi\left(-\sum_{i=1}^{n} p_{i} a_{n+1-i}+\sum_{i=1}^{n-1} \frac{1}{p_{i} a_{n-i}}\right) \\
\times\left(\prod_{i=1}^{n-1} \prod_{j=1}^{n-i}\left[a_{i}, p_{j}\right]\right) \mathrm{d} p_{n} \mathrm{~d} p_{n-1} \cdots \mathrm{d} p_{1}
\end{gathered}
$$

where the integral over $p_{i} \in F, i=1, \ldots, n$ is only iterated. Although the integrand is a priori only defined for $p_{1}, \ldots, p_{n-1} \in F^{\times}$and $p_{n} \in F$ we make sense of (5) in the proof of theorem 1.2. Denote by $w_{n} \in \mathrm{GL}_{n}(F)$ the permutation matrix with a unit anti-diagonal. For any matrix $X \in M_{n}(F)$ we will denote by $\operatorname{Tr}(X)$ the trace of $X$. For a function $\Phi \in C_{c}^{\infty}(\mathcal{S})$ let

$$
\hat{\Phi}(s)=\int_{\mathcal{S}} \Phi(t) \psi(-\operatorname{Tr}(s t)) \mathrm{d} t
$$

BULletin DE LA SOCiÉtÉ MATHÉMATIQUE DE FRANCE 
be the standard Fourier transform of $\Phi$. We will consider the Fourier transform

$$
\check{\Phi}(s)=\hat{\Phi}\left(w_{n} s w_{n}\right)
$$

of $\Phi$. Our main theorem is

THEOREM 1.2. - The integral (5) defining the Kloosterman transform on $\Omega_{\psi, n}$ is a convergent iterated integral. Moreover, let $\Phi \in C_{c}^{\infty}(\mathcal{S})$ then,

$$
\begin{aligned}
& \left(K_{\psi, n} \widetilde{\omega}^{\psi}[\Phi, \psi ; .]\right)\left(a_{1}, \ldots, a_{n}\right) \\
& \quad=|2|^{\frac{1}{2} n(n-1)} \gamma(1, \bar{\psi})^{\frac{1}{2} n(n-1)} \widetilde{\omega}^{\bar{\psi}}\left[\check{\Phi}, \bar{\psi} ; a_{1}, \ldots, a_{n}\right] .
\end{aligned}
$$

The theorem shows in particular that $K_{\psi, n}$ is a transform from $\Omega_{\psi, n}$ to $\Omega_{\bar{\psi}, n}$. Combining the theorem with Fourier inversion on $\mathcal{S}$ we obtain the inversion of the Kloosterman transform.

COROLlary 1.3. - The Kloosterman transform satisfies

$$
K_{\bar{\psi}, n} \circ K_{\psi, n}=|2|^{n(n-1)} \mathrm{Id}
$$

where Id is the identity map on $\Omega_{\psi, n}$.

The motivation to the problem lies in a conjectural trace formula identity of the Jacquet type. The identity is concerned with the metaplectic correspondence of [1]. It is a lifting of genuine automorphic representations of the metaplectic double cover $\widetilde{\mathrm{GL}}_{n}$ of $\mathrm{GL}_{n}$ to automorphic representations of $\mathrm{GL}_{n}$. Jacquet suggests the following characterization for the image of this lift: A cuspidal automorphic representation of $\mathrm{GL}_{n}$ with trivial central character is a lifting from $\widetilde{\mathrm{GL}}_{n}$ iff it is $(H, \chi)$-distinguished for some subgroup $H$ of orthogonal similitudes of $\mathrm{GL}_{n}$ and some idéle class quadratic character $\chi$.

For more detail and definitions we refer to [6]. This characterization of the image of metaplectic correspondence will follow from the relative trace formula identity

$$
\int K_{\Phi}(u) \theta\left(u^{2}\right) \mathrm{d} u=\int K_{f}\left(u_{1}, u_{2}\right) \theta\left(u_{1} u_{2}\right) \mathrm{d} u_{1} \mathrm{~d} u_{2} .
$$

Here $k$ is a global field. The integration is over $u, u_{1}, u_{2} \in N_{n}(k) \backslash N_{n}\left(\mathbb{A}_{k}\right)$, where on the right hand side $N_{n}$ is viewed as its splitting in $\widetilde{\mathrm{GL}}_{n}, K_{f}$ and $K_{\Phi}$ are kernel functions depending on the quadratic character $\chi$, of operators corresponding to the smooth functions of compact support $f$ on $\widetilde{G L}_{n}\left(\mathbb{A}_{k}\right)$ and $\Phi$ on $S_{n}\left(\mathbb{A}_{k}\right)$. Again for more details we refer to [6]. The fundamental lemma for this situation is a matching of Kloosterman integrals. If $\Phi_{0}$ is the characteristic function of $\mathcal{S} \cap K$ where $K=\operatorname{GL}_{n}\left(\mathcal{O}_{F}\right)$ is the standard maximal compact of $\operatorname{GL}_{n}(F)$, then $\omega\left[\Phi_{0}, \psi ; a\right]$ matches in an appropriate way, an integral of the form

$$
\int_{N \times N} \Psi_{0}\left({ }^{t} u_{1} a u_{2}\right) \theta\left(u_{1} u_{2}\right) \mathrm{d} u_{1} \mathrm{~d} u_{2}
$$

TOME $133-2005-\mathrm{N}^{\mathrm{O}} 3$ 
where $\Psi_{0}$ is the unit element of the genuine spherical Hecke algebra of $\widetilde{\mathrm{GL}}_{n}(F)$. In [6], Mao proved the fundamental lemma for the case $n=3$ by brute force computation. In [4], Jacquet developed a method to prove Kloosterman integral identities of the above type. The method requires in both sides an inversion formula for a Fourier-Kloosterman transform on the space of Kloosterman integrals. For the case of a quadratic extension the inversion formulas are obtained in [3] and the method is carried out in [4] to prove the identity of Kloosterman integrals which serves as a fundamental lemma for a relative trace formula. In this work we provide a step towards the fundamental lemma associated with the trace formula (9). At the same time, the formula (5) and mainly the oscillating factor $\prod_{i=1}^{n-1} \prod_{j=1}^{n-i}\left[a_{i}, p_{j}\right]$ in it, hint to a relation with the metaplectic group. If $\sigma$ is the 2-cocycle that defines multiplication in $\widetilde{\mathrm{GL}}_{n}(F)$ as defined in [5], then for $a=\operatorname{diag}\left(a_{1}, \ldots, a_{n}\right)$ and $p=\operatorname{diag}\left(p_{1}, \ldots, p_{n}\right)$ we have

$$
\sigma\left(a, w_{n} p w_{n}\right)=\prod_{i=1}^{n-1} \prod_{j=1}^{n-i}\left[a_{i}, p_{j}\right]
$$

The rest of this manuscript is organized as follows: The main tool we use to prove Theorem 1.2 is Weil's formula. In Chapter 2 we write it in a form convenient for our needs. We then prove the theorem by induction. Chapter 3 provides an inversion formula for some intermediate integrals designed to use an inductive argument. In Chapter 4 the inductive step is carried out to finish the proof of the inversion. Chapter 5 provides a much simpler formula associated with the smallest orbits. We present it here, since once the analogous results for the metaplectic case will be obtained, the method of Jacquet requires this formula in order to prove smooth matching. The proof of the inversion formula closely follows the guidelines of [3], the new ingredient is the occurrence of the Hilbert symbol in the Kloosterman transform. The problem was suggested to me by Jacquet. For the project and for much help and support, I am most thankful to him. Most of this work was written during my visit at IHÉS. I thank the IHÉS for a very pleasant and productive visit.

\section{Weil's formula}

Let

$$
V_{n, m}=\left\{\left(\begin{array}{cc}
0_{n} & X \\
{ }^{t} X & 0_{m}
\end{array}\right) ; X \in M_{n \times m}(F)\right\} .
$$

We view $V_{n, m}$ as a self-dual space via the pairing

$$
\left(\left(\begin{array}{cc}
0_{n} & X \\
{ }^{t} X & 0_{m}
\end{array}\right),\left(\begin{array}{cc}
0_{n} & { }^{t} Y \\
Y & 0_{m}
\end{array}\right)\right) \longmapsto \operatorname{Tr}\left[-\left(\begin{array}{cc}
0_{n} & X \\
{ }^{t} X & 0_{m}
\end{array}\right)\left(\begin{array}{cc}
0_{n} & { }^{t} Y \\
Y & 0_{m}
\end{array}\right)\right]
$$

BULletin DE LA SOCiÉtÉ MATHÉMATIQUE DE FRANCE 
where $X \in M_{n \times m}(F)$ and $Y \in M_{m \times n}(F)$. The Fourier transform of a function $\Phi \in C_{c}^{\infty}\left(V_{n, m}\right)$ is defined by

$$
\hat{\Phi}\left(\begin{array}{cc}
0_{n} & { }^{t} X \\
X & 0_{m}
\end{array}\right)=\int_{M_{n \times m}} \Phi\left(\begin{array}{cc}
0_{n} & Y \\
{ }^{t} Y & 0_{m}
\end{array}\right) \psi[-2 \operatorname{Tr}(X Y)] \mathrm{d} Y,
$$

$X \in M_{m \times n}(F)$. We recall Weil's formula for the Fourier transform of a character of second order for the space $V_{n, m}$. Taking our definition of the Fourier transform into acount, from [7] we have that for all $A \in S_{n}, C \in S_{m}$ there is a constant $\gamma_{m}^{n}(A, C, \psi)$ such that for all $\Phi \in C_{c}^{\infty}\left(V_{n, m}\right)$

$$
\begin{aligned}
\int_{M_{n \times m}(F)} & \Phi\left(\begin{array}{cc}
0_{n} & X \\
{ }^{t} X & 0_{m}
\end{array}\right) \psi\left[\operatorname{Tr}\left(C^{t} X A X\right)\right] \mathrm{d} X \\
= & |2|^{\frac{1}{2} m n} \cdot|\operatorname{det} A|^{-\frac{1}{2} m} \cdot|\operatorname{det} C|^{-\frac{1}{2} n} \\
& \times \gamma_{m}^{n}(A, C, \psi) \int_{M_{m \times n}(F)} \hat{\Phi}\left(\begin{array}{cc}
0_{n}{ }^{t} Z \\
Z & 0_{m}
\end{array}\right) \psi\left[-\operatorname{Tr}\left(C^{-1} Z A^{-1 t} Z\right)\right] \mathrm{d} Z .
\end{aligned}
$$

For $P \in M_{m \times n}(F)$ the Fourier transform of the function

$$
\left(\begin{array}{cc}
0_{n} & X \\
{ }^{t} X & 0_{m}
\end{array}\right) \longmapsto \Phi\left[\left(\begin{array}{cc}
0_{n} & X \\
{ }^{t} X & 0_{m}
\end{array}\right)\right] \psi[\operatorname{Tr}(P X)]
$$

$X \in M_{n \times m}(F)$, is the function

$$
\left(\begin{array}{cc}
0_{n}{ }^{t} Z \\
Z & 0_{m}
\end{array}\right) \longmapsto \hat{\Phi}\left(\begin{array}{cc}
0_{n} & { }^{t}\left(Z-\frac{1}{2} P\right) \\
Z-\frac{1}{2} P & 0_{m}
\end{array}\right)
$$

$Z \in M_{m \times n}(F)$. Applying Weil's formula (14) to this function, we get after the change of variables $Z \mapsto Z+\frac{1}{2} P$ that

$$
\begin{aligned}
& \int_{M_{n \times m}(F)} \Phi\left[\left(\begin{array}{cc}
0_{n} & X \\
{ }^{t} X & 0_{m}
\end{array}\right)\right] \psi[\left.\operatorname{Tr}(P X)+\operatorname{Tr}\left(C^{t} X A X\right)\right] \mathrm{d} X \\
&=|2|^{\frac{1}{2} m n}|\operatorname{det} A|^{-\frac{1}{2} m} \cdot|\operatorname{det} C|^{-\frac{1}{2} n} \gamma_{m}^{n}(A, C, \psi) \int_{M_{m \times n}(F)} \hat{\Phi}\left(\begin{array}{cc}
0_{n} & { }^{t} Z \\
Z & 0_{m}
\end{array}\right) \\
& \times \psi\left[-\operatorname{Tr}\left(C^{-1}\left(Z+\frac{1}{2} P\right) A^{-1 t}\left(Z+\frac{1}{2} P\right)\right)\right] \mathrm{d} Z
\end{aligned}
$$

We remark that in the case $m=n=1$ the Weil constant is the 1-dimensional Weil constant defined in (1)

$$
\gamma_{1}^{1}(A, C, \psi)=\gamma(A C, \psi) .
$$

For general $m$ and $n$ we can describe the Weil constant in terms of the 1-dimensional case.

LEMmA 2.1. - Let $A={ }^{t} g_{1} \operatorname{diag}\left(a_{1}, \ldots, a_{n}\right) g_{1}$ and $C=g_{2} \operatorname{diag}\left(c_{1}, \ldots, c_{m}\right)^{t} g_{2}$, where $A \in S_{n}, C \in S_{m}, g_{1} \in \mathrm{GL}_{n}(F)$ and $g_{2} \in \mathrm{GL}_{m}(F)$, then

$$
\gamma_{m}^{n}(A, C, \psi)=\prod_{\substack{1 \leq i \leq n \\ 1 \leq j \leq m}} \gamma\left(a_{i} c_{j}, \psi\right)
$$

TOME $133-2005-\mathrm{N}^{\mathrm{O}} 3$ 
Proof. - We start with the case $m=1$. Since $C_{c}^{\infty}\left(V_{n, 1}\right) \simeq C_{c}^{\infty}(F)^{\otimes n}$, it is enough in the definition of $\gamma_{1}^{n}$ to consider functions $\Phi$ of the form $\Phi(v)=$ $\prod_{i=1}^{n} f_{i}\left(x_{i}\right)$ where $v \in V_{n, 1}$ is of the form $v=\left(\begin{array}{cc}0_{n} & X \\ { }^{t} X & 0\end{array}\right), X={ }^{t}\left(x_{1}, \ldots, x_{n}\right) \in F^{n}$ and $f_{i} \in C_{c}^{\infty}(F)$. Note that in this case $\hat{\Phi}(v)=\prod_{i=1}^{n} \hat{f}_{i}\left(x_{i}\right)$, therefore if $A=\operatorname{diag}\left(a_{1}, \ldots, a_{n}\right)$ the factorization of $\gamma_{1}^{n}(A, 1, \psi)$ is straightforward from (1) and (2). Indeed

$$
\begin{gathered}
\int \Phi\left(\begin{array}{cc}
0_{n} & X \\
{ }^{t} X & 0
\end{array}\right) \psi\left[\operatorname{Tr}\left({ }^{t} X A X\right] \mathrm{d} X=\prod_{i=1}^{n} \int f_{i}\left(x_{i}\right) \psi\left(a_{i} x_{i}^{2}\right) \mathrm{d} x_{i}\right. \\
=|2|^{\frac{1}{2} n} \prod_{i=1}^{n}\left(\left|a_{i}\right|^{-\frac{1}{2}} \gamma\left(a_{i}, \psi\right) \int \hat{f}_{i}\left(x_{i}\right) \psi\left(-a_{i}^{-1} x_{i}^{2}\right) \mathrm{d} x_{i}\right) \\
=|2|^{\frac{1}{2} n}|\operatorname{det} A|^{-\frac{1}{2}}\left(\prod_{i=1}^{n} \gamma\left(a_{i}, \psi\right)\right) \int \hat{\Phi}\left(\begin{array}{cc}
0_{n} & { }^{t} Z \\
Z & 0
\end{array}\right) \psi\left[-\operatorname{Tr}\left(Z A^{-1 t} Z\right)\right] \mathrm{d} Z,
\end{gathered}
$$

therefore in this case indeed we get

$$
\gamma_{1}^{n}(A, 1, \psi)=\prod_{i=1}^{n} \gamma\left(a_{i}, \psi\right) .
$$

Let $g \in \mathrm{GL}_{n}(F)$ be such that $A={ }^{t} g a g$ with $a=\operatorname{diag}\left(a_{1}, \ldots, a_{n}\right)$, for any $A \in S_{n}$. Note that the Fourier transform of the function

$$
\left(\begin{array}{cc}
0_{n} & X \\
{ }^{t} X & 0_{m}
\end{array}\right) \longmapsto \Phi\left(\begin{array}{cc}
0_{n} & g^{-1} X \\
{ }^{t} X^{t} g^{-1} & 0
\end{array}\right)
$$

is the function

$$
\left(\begin{array}{cc}
0_{n} & { }^{t} Z \\
Z & 0_{m}
\end{array}\right) \longmapsto|\operatorname{det} g| \cdot \hat{\Phi}\left(\begin{array}{cc}
0_{n} & { }^{t} g^{t} Z \\
Z g & 0
\end{array}\right)
$$

and therefore using the changes of variables $X \mapsto g^{-1} X, Z \mapsto Z g^{-1}$ and applying (20) for $a$ we get

$$
\begin{array}{r}
\int \Phi\left(\begin{array}{cc}
0_{n} & X \\
{ }^{t} X & 0
\end{array}\right) \psi\left[\operatorname{Tr}\left({ }^{t} X A X\right)\right] \mathrm{d} X=|\operatorname{det} g|^{-1} \int \Phi\left(\begin{array}{cc}
0_{n} & g^{-1} X \\
{ }^{t} X^{t} g^{-1} & 0
\end{array}\right) \psi\left[\operatorname{Tr}\left({ }^{t} X a X\right)\right] \mathrm{d} X \\
=|2|^{\frac{1}{2} n}|\operatorname{det} a|^{-\frac{1}{2}}\left(\prod_{i=1}^{n} \gamma\left(a_{i}, \psi\right)\right) \int \hat{\Phi}\left(\begin{array}{cc}
0_{n} & { }^{t} g^{t} Z \\
Z g & 0
\end{array}\right) \psi\left[-\operatorname{Tr}\left(Z a^{-1 t} Z\right)\right] \mathrm{d} Z \\
=|2|^{\frac{1}{2} n}|\operatorname{det} g|^{-1} \cdot|\operatorname{det} a|^{-\frac{1}{2}}\left(\prod_{i=1}^{n} \gamma\left(a_{i}, \psi\right)\right) \int \hat{\Phi}\left(\begin{array}{cc}
0_{n}{ }^{t} Z \\
Z & 0
\end{array}\right) \psi\left[-\operatorname{Tr}\left(Z A^{-1 t} Z\right)\right] \mathrm{d} Z .
\end{array}
$$

Since $\operatorname{det} A=\operatorname{det} g^{2} \operatorname{det} a$ we get (20) for any $A \in S_{n}$. For a general $m$, if $C=\operatorname{diag}\left(c_{1}, \ldots, c_{m}\right)$ is diagonal then

$$
\operatorname{Tr}\left(C^{t} X A X\right)=\sum_{j=1}^{m} \operatorname{Tr}\left({ }^{t} X c_{j} A X\right) .
$$

BULLETIN DE LA SOCIÉTÉ MATHÉMATIQUe DE FRANCE 
Therefore, as in the case $m=1$ the fact that $C_{c}^{\infty}\left(V_{n, m}\right) \simeq C_{c}^{\infty}\left(V_{n, 1}\right)^{\otimes m}$ implies that

$$
\gamma_{m}^{n}(A, C, \psi)=\prod_{j=1}^{m} \gamma_{1}^{n}\left(c_{j} A, 1, \psi\right)
$$

and (19) then follows from the case $m=1$. For any $A$ and $C$ as in the statement of the lemma, since

$$
\operatorname{Tr}\left(C^{t} X A X\right)=\operatorname{Tr}\left(X C^{t} X A\right)
$$

we may compute as before, $\operatorname{denoting} c=\operatorname{diag}\left(c_{1}, \ldots, c_{m}\right)$ and using the changes of variables $X \mapsto X g_{2}^{-1}$ and $Z \mapsto g_{2}^{-1} Z$ that

$$
\begin{gathered}
\int \Phi\left(\begin{array}{cc}
0_{n} & X \\
{ }^{t} X & 0
\end{array}\right) \psi\left[\operatorname{Tr}\left(C^{t} X A X\right)\right] \mathrm{d} X \\
=\left|\operatorname{det} g_{2}\right|^{-n} \int \Phi\left(\begin{array}{cc}
0_{n} & X g_{2}^{-1} \\
{ }^{-1}{ }_{2}{ }^{t} X & 0
\end{array}\right) \psi\left[\operatorname{Tr}\left(c^{t} X A X\right)\right] \mathrm{d} X \\
=|2|^{\frac{1}{2} m n} \cdot|\operatorname{det} A|^{-\frac{1}{2} m} \cdot|\operatorname{det} c|^{-\frac{1}{2} n} \cdot \gamma_{m}^{n}(A, c, \psi) \\
\int \hat{\Phi}\left(\begin{array}{cc}
0_{n} & { }^{t} Z^{t} g_{2} \\
g_{2} Z & 0
\end{array}\right) \psi\left[-\operatorname{Tr}\left(c^{-1} Z A^{-1 t} Z\right)\right] \mathrm{d} Z \\
=|2|^{\frac{1}{2} m n} \cdot\left|\operatorname{det} g_{2}\right|^{-n} \cdot|\operatorname{det} A|^{-\frac{1}{2} m} \cdot|\operatorname{det} c|^{-\frac{1}{2} n} \cdot \gamma_{m}^{n}(A, c, \psi) \\
\int \hat{\Phi}\left(\begin{array}{cc}
0_{n}{ }^{t} Z \\
Z & 0
\end{array}\right) \psi\left[-\operatorname{Tr}\left(C^{-1} Z A^{-1 t} Z\right)\right] \mathrm{d} Z
\end{gathered}
$$

We then have

$$
\gamma_{m}^{n}(A, C, \psi)=\gamma_{m}^{n}(A, c, \psi)
$$

and the lemma follows from the case when $C$ is diagonal.

The symmetry on the right hand side of (19) implies that

$$
\gamma_{m}^{n}(A, C, \psi)=\gamma_{n}^{m}(C, A, \psi) .
$$

From (1) it is easy to see that the one-dimensional Weil constant satisfies

$$
\gamma(a, \psi)=\gamma(-a, \bar{\psi}) \text {. }
$$

Applying Weil's formula twice we get that

$$
\gamma(a, \psi) \gamma\left(-a^{-1}, \psi\right)=1
$$

We also recall that $\gamma$ satisfies

$$
\gamma(a c, \psi)=\gamma(1, \psi)^{-1} \gamma(a, \psi) \gamma(c, \psi)[a, c]
$$

for $a, c \in F^{\times}$. Therefore, for $A$ and $C$ as in Lemma 2.1

(26) $\gamma_{m}^{n}(A, C, \psi)=\gamma(1, \psi)^{-m n}\left(\prod_{i=1}^{n} \gamma\left(a_{i}, \psi\right)^{m}\right)\left(\prod_{j=1}^{m} \gamma\left(c_{j}, \psi\right)^{n}\right)\left(\prod_{i, j}\left[a_{i}, c_{j}\right]\right)$.

TOME $133-2005-\mathrm{N}^{\mathrm{O}} 3$ 
In particular

$$
\gamma_{m}^{n}\left(A, 1_{m}, \psi\right)=\prod_{i=1}^{n} \gamma\left(a_{i}, \psi\right)^{m}=\gamma_{n}^{m}\left(1_{m}, A, \psi\right)
$$

and

$$
\gamma_{n}^{m}\left(1_{n}, C, \psi\right)=\prod_{j=1}^{m} \gamma\left(c_{j}, \psi\right)^{n}=\gamma_{m}^{n}\left(C, 1_{n}, \psi\right) .
$$

The expression $\prod_{i, j}\left[a_{i}, c_{j}\right]$ is thus only dependent on $A$ and $C$ and we can and will denote

$$
[A, C]=\prod_{i, j}\left[a_{i}, c_{j}\right]
$$

We finish this chapter, with an identity we will need later.

LEMma 2.2. - One has

$$
\gamma_{m}^{n}\left(-A, 1_{m}, \psi\right) \gamma_{m}^{n}\left(A^{-1}, C, \psi\right)=\gamma(1, \bar{\psi})^{m n}[A, C] \gamma_{n}^{m}\left(-C, 1_{n}, \bar{\psi}\right)
$$

Proof. - From (26), (27) and (28) we get that

(31) $\gamma_{m}^{n}\left(-A, 1_{m}, \psi\right) \gamma_{m}^{n}\left(A^{-1}, C, \psi\right)$

$$
=\gamma(1, \psi)^{-m n} \gamma_{m}^{n}\left(-A, 1_{m}, \psi\right) \gamma_{m}^{n}\left(A^{-1}, 1_{m}, \psi\right) \gamma_{n}^{m}\left(C, 1_{n}, \psi\right)[A, C] .
$$

Using (24) and (27) we get that

$$
\gamma_{m}^{n}\left(-A, 1_{m}, \psi\right) \gamma_{m}^{n}\left(A^{-1}, 1_{m}, \psi\right)=1
$$

From (23) and (28) we have $\gamma_{n}^{m}\left(C, 1_{n}, \psi\right)=\gamma_{n}^{m}\left(-C, 1_{n}, \bar{\psi}\right)$ and from (23) and $(24)$ that $\gamma(1, \psi)^{-1}=\gamma(1, \bar{\psi})$. Therefore, the right hand side of (31) is now equal to $\gamma(1, \bar{\psi})^{m n}[A, C] \gamma_{n}^{m}\left(-C, 1_{n}, \bar{\psi}\right)$ and the lemma follows.

\section{Intermediate orbital integrals}

For $n, m \geq 1$ and a function $\Phi \in C_{c}^{\infty}\left(\mathcal{S}_{m+n}\right)$ we define the intermediate orbital integral

$$
\begin{aligned}
& \omega_{m}^{n}\left[\Phi, \psi ;\left(\begin{array}{ll}
A_{n} & \\
& B_{m}
\end{array}\right)\right] \\
& \quad=\int_{M_{n \times m}(F)} \Phi\left[\left(\begin{array}{ll}
1_{n} & \\
t X & 1_{m}
\end{array}\right)\left(\begin{array}{cc}
A_{n} & \\
& B_{m}
\end{array}\right)\left(\begin{array}{cc}
1_{n} & X \\
& 1_{m}
\end{array}\right)\right] \theta_{m+n}\left[\left(\begin{array}{cc}
1_{n} & 2 X \\
& 1_{m}
\end{array}\right)\right] \mathrm{d} X \\
& =\int_{M_{n \times m}(F)} \Phi\left[\left(\begin{array}{cc}
A_{n} & A_{n} X \\
{ }^{t} X A_{n} & B_{m}+{ }^{t} X A_{n} X
\end{array}\right)\right] \psi[2 \operatorname{Tr}(\epsilon X)] \mathrm{d} X
\end{aligned}
$$

BULletin DE LA SOCiÉtÉ MATHÉMATIQUe DE FRANCE 
where $A_{n} \in S_{n}, B_{m} \in \mathcal{S}_{m}$ and $\epsilon=\epsilon_{m}^{n}=\left(\delta_{(i, j),(1, n)}\right) \in M_{m \times n}(F)$. We also define a normalized intermediate integral

$$
\begin{aligned}
\widetilde{\omega}_{m}^{n, \psi}[\Phi, \psi ; & \left.\left(\begin{array}{ll}
A_{n} & \\
& B_{m}
\end{array}\right)\right] \\
& =\gamma_{m}^{n}\left(-A_{n}, 1_{m}, \psi\right) \cdot\left|\operatorname{det} A_{n}\right|^{\frac{1}{2} m} \cdot \omega_{m}^{n}\left[\Phi, \psi ;\left(\begin{array}{ll}
A_{n} & \\
& B_{m}
\end{array}\right)\right] .
\end{aligned}
$$

Proposition 3.1. - Let $C_{m} \in S_{m}, D_{n} \in \mathcal{S}_{n}$ and $\Phi \in C_{c}^{\infty}\left(\mathcal{S}_{m+n}\right)$. The function mapping $A_{n}$ to

$$
\begin{aligned}
& {\left[A_{n}, C_{m}\right] \psi\left[\operatorname{Tr}\left(w_{m} C_{m}^{-1} w_{m} \epsilon_{m}^{n} A_{n}^{-1 t} \epsilon_{m}^{n}\right)\right]} \\
& \quad \times \int_{\mathcal{S}_{m}} \widetilde{\omega}_{m}^{n, \psi}\left[\Phi, \psi ;\left(\begin{array}{cc}
A_{n} & \\
& B_{m}
\end{array}\right)\right] \psi\left[-\operatorname{Tr}\left(B_{m} w_{m} C_{m} w_{m}\right)\right] \mathrm{d} B_{m}
\end{aligned}
$$

originally defined for $A_{n} \in S_{n}$, extends to a smooth function of compact support on $\mathcal{S}_{n}$. The iterated integral

$$
\begin{aligned}
\int_{\mathcal{S}_{n}}\{ & \left.\int_{\mathcal{S}_{m}} \widetilde{\omega}_{m}^{n, \psi}\left[\Phi, \psi ;\left(\begin{array}{cc}
A_{n} & \\
& B_{m}
\end{array}\right)\right] \psi\left[-\operatorname{Tr}\left(B_{m} w_{m} C_{m} w_{m}\right)\right] \mathrm{d} B_{m}\right\} \\
& \times\left[A_{n}, C_{m}\right] \psi\left[\operatorname{Tr}\left(w_{m} C_{m}^{-1} w_{m} \epsilon_{m}^{n} A_{n}^{-1 t} \epsilon_{m}^{n}\right)\right] \psi\left[-\operatorname{Tr}\left(A_{n} w_{n} D_{n} w_{n}\right)\right] \mathrm{d} A_{n}
\end{aligned}
$$

is therefore convergent. It is equal to

$$
|2|^{\frac{1}{2} m n} \cdot \gamma(1, \bar{\psi})^{m n} \cdot \widetilde{\omega}_{n}^{m, \bar{\psi}}\left[\check{\Phi}, \bar{\psi} ;\left(\begin{array}{cc}
C_{m} & \\
& D_{n}
\end{array}\right)\right] .
$$

Proof. - First we remark that from the right hand side of (32) it is easily observed that for a fixed $A_{n} \in S_{n}$ the function

$$
B_{m} \longmapsto \omega_{m}^{n}\left[\Phi, \psi ;\left(\begin{array}{ll}
A_{n} & \\
& B_{m}
\end{array}\right)\right]
$$

is smooth and of compact support on $\mathcal{S}_{m}$. Therefore, for a fixed $C_{m} \in S_{m}$ the function $\Theta$ defined by

$$
\Theta\left(D_{n}\right)=|2|^{\frac{1}{2} m n} \gamma(1, \bar{\psi})^{m n} \cdot \widetilde{\omega}_{n}^{m, \bar{\psi}}\left[\check{\Phi}, \bar{\psi} ;\left(\begin{array}{l}
w_{m} C_{m} w_{m} \\
w_{n} D_{n} w_{n}
\end{array}\right)\right],
$$

is in $C_{c}^{\infty}\left(\mathcal{S}_{n}\right)$. Consider the partial Fourier transform with respect to $B_{m}$

$$
\int_{\mathcal{S}_{m}} \widetilde{\omega}_{m}^{n}\left[\Phi, \psi ;\left(\begin{array}{ll}
A_{n} & \\
& B_{m}
\end{array}\right)\right] \psi\left[\operatorname{Tr}\left(-C_{m} B_{m}\right)\right] \mathrm{d} B_{m} .
$$

Expanding along (32) it becomes after a change of variables $B_{m} \mapsto B_{m}-{ }^{t} X A_{n} X$ (39) $\gamma_{m}^{n}\left(-A_{n}, 1_{m}, \psi\right) \cdot\left|\operatorname{det} A_{n}\right|^{\frac{1}{2} m}$.

$$
\times \int \Phi\left[\left(\begin{array}{cc}
A_{n} & A_{n} X \\
{ }^{t} X A_{n} & B_{m}
\end{array}\right)\right] \psi\left[\operatorname{Tr}\left(2 \epsilon_{m}^{n} X\right)+\begin{array}{r}
\left.\operatorname{Tr}\left(C_{m}{ }^{t} X A_{n} X\right)\right] \mathrm{d} X \\
\psi\left[\operatorname{Tr}\left(-C_{m} B_{m}\right)\right] \mathrm{d} B_{m}
\end{array}\right.
$$

TOME $133-2005-\mathrm{N}^{\mathrm{O}} 3$ 
and after another change of variables $X \mapsto A_{n}^{-1} X$ it becomes

(40) $\gamma_{m}^{n}\left(-A_{n}, 1_{m}, \psi\right)\left|\operatorname{det} A_{n}\right|^{-\frac{1}{2} m}$

$$
\begin{array}{r}
\int \Phi\left[\left(\begin{array}{cc}
A_{n} & X \\
{ }^{t} X & B_{m}
\end{array}\right)\right] \psi\left[\operatorname{Tr}\left(2 \epsilon_{m}^{n} A_{n}^{-1} X\right)+\operatorname{Tr}\left(C_{m}{ }^{t} X A_{n}^{-1} X\right)\right] \mathrm{d} X \\
\psi\left[-\operatorname{Tr}\left(B_{m} C_{m}\right)\right] \mathrm{d} B_{m}
\end{array}
$$

Applying Weil's formula (17) with $P=2 \epsilon_{m}^{n} A_{n}^{-1}$, we see that this is equal to

$$
\begin{gathered}
|2|^{\frac{1}{2} m n} \gamma_{m}^{n}\left(A_{n}^{-1}, C_{m}, \psi\right) \gamma_{m}^{n}\left(-A_{n}, 1_{m}, \psi\right)\left|\operatorname{det} C_{m}\right|^{-\frac{1}{2} n} \\
\times \int \Phi\left(\begin{array}{cc}
A_{n} & Y \\
{ }^{t} Y & B_{m}
\end{array}\right) \psi[-2 \operatorname{Tr}(X Y)] \mathrm{d} Y \\
\psi\left[-\operatorname{Tr}\left(C_{m}^{-1}\left(X+\epsilon_{m}^{n} A_{n}^{-1}\right) A_{n}\left({ }^{t} X+A_{n}^{-1 t} \epsilon_{m}^{n}\right)\right)\right] \mathrm{d} X \\
\psi\left[-\operatorname{Tr}\left(B_{m} C_{m}\right)\right] \mathrm{d} B_{m} .
\end{gathered}
$$

We now use Lemma 2.2. After expanding (41) it becomes:

$$
\begin{aligned}
|2|^{\frac{1}{2} m n} & \cdot \gamma(1, \bar{\psi})^{m n} \cdot \gamma_{n}^{m}\left(-C_{m}, 1_{n}, \bar{\psi}\right) \cdot\left|\operatorname{det} C_{m}\right|^{-\frac{1}{2} n} \\
\psi[ & \left.-\operatorname{Tr}\left(C_{m}^{-1} \epsilon_{m}^{n} A_{n}^{-1 t} \epsilon_{m}^{n}\right)\right]\left[A_{n}, C_{m}\right] \\
& \times \int \Phi\left(\begin{array}{cc}
A_{n} & Y \\
{ }^{t} Y & B_{m}
\end{array}\right) \psi\left[-2 \operatorname{Tr}(X Y)-\operatorname{Tr}\left(C_{m}^{-1} X A_{n}{ }^{t} X\right)\right. \\
& \left.\quad-2 \operatorname{Tr}\left(C_{m}^{-1} X^{t} \epsilon_{m}^{n}\right)\right] \mathrm{d} Y \mathrm{~d} X \cdot \psi\left[-\operatorname{Tr}\left(B_{m} C_{m}\right)\right] \mathrm{d} B_{m}
\end{aligned}
$$

We showed so far that for a fixed $C_{m} \in S_{m}$, the function $\Psi$ on $S_{n}$ defined by (43) $\Psi\left(A_{n}\right)=\left[A_{n}, C_{m}\right] \cdot \psi\left[\operatorname{Tr}\left(C_{m}^{-1} \epsilon_{m}^{n} A_{n}^{-1} \epsilon_{n}^{m}\right)\right] \cdot$

$$
\times \int_{\mathcal{S}_{m}} \widetilde{\omega}_{m}^{n, \psi}\left[\Phi, \psi ;\left(\begin{array}{cc}
A_{n} & \\
& B_{m}
\end{array}\right)\right] \psi\left[-\operatorname{Tr}\left(B_{m} C_{m}\right)\right] \mathrm{d} B_{m}
$$

is equal to

$$
\begin{aligned}
& |2|^{\frac{1}{2} m n} \cdot \gamma(1, \bar{\psi})^{m n} \cdot \gamma_{n}^{m}\left(-C_{m}, 1_{n}, \bar{\psi}\right) \cdot\left|\operatorname{det} C_{m}\right|^{-\frac{1}{2} n} \\
& \int \Phi\left(\begin{array}{cc}
A_{n} & Y \\
{ }^{t} Y & B_{m}
\end{array}\right) \psi\left[-2 \operatorname{Tr}(X Y)-\operatorname{Tr}\left(C_{m}^{-1} X A_{n}{ }^{t} X\right)\right. \\
& \left.\quad-2 \operatorname{Tr}\left(C_{m}^{-1} X^{t} \epsilon_{m}^{n}\right)-\operatorname{Tr}\left(C_{m} B_{m}\right)\right] \mathrm{d} Y \mathrm{~d} X \mathrm{~d} B_{m}
\end{aligned}
$$

Changing variables $X \mapsto C_{m} X$ we get

$$
\begin{aligned}
& \Psi\left(A_{n}\right)=|2|^{\frac{1}{2} m n} \cdot \gamma(1, \bar{\psi})^{m n} \cdot \gamma_{n}^{m}\left(-C_{m}, 1_{n}, \bar{\psi}\right) \cdot\left|\operatorname{det} C_{m}\right|^{\frac{1}{2} n} \\
& \times \int \Phi\left(\begin{array}{cc}
A_{n} & Y \\
{ }^{t} Y & B_{m}
\end{array}\right) \cdot \psi\left[-2 \operatorname{Tr}\left(C_{m} X Y\right)-\operatorname{Tr}\left(X A_{n}{ }^{t} X C_{m}\right)\right. \\
& \left.-2 \operatorname{Tr}\left(X^{t} \epsilon_{m}^{n}\right)-\operatorname{Tr}\left(B_{m} C_{m}\right)\right] \mathrm{d} Y \mathrm{~d} X \mathrm{~d} B_{m} .
\end{aligned}
$$

BULLETIN DE LA SOCiÉTÉ MATHÉMATIQUe DE FRANCE 
Next we expand $\Theta\left(D_{n}\right)$ :

$$
\begin{aligned}
& \text { (46) } \Theta\left(D_{n}\right)=|2|^{\frac{1}{2} m n} \cdot \gamma(1, \bar{\psi})^{m n} \cdot \gamma_{n}^{m}\left(-C_{m}, 1_{n}, \bar{\psi}\right) \cdot\left|\operatorname{det} C_{m}\right|^{\frac{1}{2} n} \\
& \times \int_{M_{m \times n}(F)} \hat{\Phi}\left[w_{m+n}\left(\begin{array}{cc}
w_{m} C_{m} w_{m} & w_{m} C_{m} w_{m} X \\
{ }^{t} X w_{m} C_{m} w_{m} & w_{n} D_{n} w_{n}+{ }^{t} X w_{m} C_{m} w_{m} X
\end{array}\right) w_{m+n}\right] \\
&=|2|^{\frac{1}{2} m n} \gamma(1, \bar{\psi})^{m n} \cdot \gamma_{n}^{m}\left(-C_{m}, 1_{n}, \bar{\psi}\right) \cdot\left|\operatorname{det} C_{m}\right|^{\frac{1}{2} n} \psi\left[-2 \operatorname{Tr}\left(\epsilon_{n}^{m} X\right)\right] \mathrm{d} X \\
& \times \int_{M_{m \times n}(F)} \hat{\Phi}\left[\left(\begin{array}{cc}
D_{n}+w_{n}{ }^{t} X w_{m} C_{m} w_{m} X w_{n} & w_{n}{ }^{t} X w_{m} C_{m} \\
C_{m} w_{m} X w_{n} & C_{m}
\end{array}\right)\right] \\
&
\end{aligned}
$$

After a change of variables $X \mapsto w_{m} X w_{n}, \Theta\left(D_{n}\right)$ becomes

$$
\begin{aligned}
& |2|^{\frac{1}{2} m n} \cdot \gamma(1, \bar{\psi})^{m n} \cdot \gamma_{n}^{m}\left(-C_{m}, 1_{n}, \bar{\psi}\right) \cdot\left|\operatorname{det} C_{m}\right|^{\frac{1}{2} n} \cdot \\
& \quad \times \int_{M_{m \times n}(F)} \hat{\Phi}\left[\left(\begin{array}{cc}
D_{n}+{ }^{t} X C_{m} X & { }^{t} X C_{m} \\
C_{m} X & C_{m}
\end{array}\right)\right] \psi\left[-2 \operatorname{Tr}\left(X^{t} \epsilon_{m}^{n}\right)\right] \mathrm{d} X
\end{aligned}
$$

where we use the fact that

$$
\operatorname{Tr}\left(\epsilon_{n}^{m} w_{m} X w_{n}\right)=X_{1, n}=\operatorname{Tr}\left(X^{t} \epsilon_{m}^{n}\right) .
$$

Expanding further we have

(48) $\Theta\left(D_{n}\right)=|2|^{\frac{1}{2} m n} \cdot \gamma(1, \bar{\psi})^{m n} \cdot \gamma_{n}^{m}\left(-C_{m}, 1_{n}, \bar{\psi}\right) \cdot\left|\operatorname{det} C_{m}\right|^{\frac{1}{2} n} \cdot$

$$
\begin{aligned}
& \times \int \Phi\left[\left(\begin{array}{cc}
s_{n} & Y \\
{ }^{t} Y & B_{m}
\end{array}\right)\right] \psi\left[-\operatorname{Tr}\left(\left(\begin{array}{cc}
s_{n} & Y \\
{ }^{t} Y & B_{m}
\end{array}\right)\left(\begin{array}{cc}
D_{n}+{ }^{t} X C_{m} X & { }^{t} X C_{m} \\
C_{m} X & C_{m}
\end{array}\right)\right)\right] \\
= & \mathrm{d} Y \mathrm{~d} s_{n} \mathrm{~d} B_{m} \psi\left[-2 \operatorname{Tr}\left(X^{t} \epsilon_{m}^{n}\right)\right] \mathrm{d} X \\
& \times \int \Phi\left[\left(\begin{array}{cc}
s_{n} & Y \\
{ }^{t} Y & B_{m}
\end{array}\right)\right] \psi\left[-\operatorname{Tr}\left(s_{n}{ }^{t} X C_{m} X\right)-\operatorname{Tr}\left(s_{n} D_{n}\right)\right] \mathrm{d} s_{n} \\
& \psi\left[-\operatorname{Tr}\left(B_{m} C_{m}\right)-2 \operatorname{Tr}\left(C_{m} X Y\right)\right] \mathrm{d} Y \mathrm{~d} B_{m} \psi\left[-2 \operatorname{Tr}\left(X^{t} \epsilon_{m}^{n}\right)\right] \mathrm{d} X .
\end{aligned}
$$

Using Fourier inversion we then see that

$$
\begin{array}{r}
\widehat{\Theta}\left(-A_{n}\right)=\int_{\mathcal{S}_{n}} \Theta\left(D_{n}\right) \psi\left[\operatorname{Tr}\left(A_{n} D_{n}\right)\right] \mathrm{d} A_{n} \\
=|2|^{\frac{1}{2} m n} \gamma(1, \bar{\psi})^{m n} \gamma_{n}^{m}\left(-C_{m}, 1_{n}, \bar{\psi}\right)\left|\operatorname{det} C_{m}\right|^{\frac{1}{2} n} \\
\times \int \Phi\left[\left(\begin{array}{cc}
A_{n} & Y \\
{ }^{t} Y & B_{m}
\end{array}\right)\right] \psi\left[-\operatorname{Tr}\left(A_{n}{ }^{t} X C_{m} X\right)\right] \\
\psi\left[-\operatorname{Tr}\left(B_{m} C_{m}\right)-2 \operatorname{Tr}\left(C_{m} X Y\right)\right] \mathrm{d} Y \mathrm{~d} B_{m} \\
\psi\left[-2 \operatorname{Tr}\left(X^{t} \epsilon_{m}^{n}\right)\right] \mathrm{d} X .
\end{array}
$$

TOME $133-2005-\mathrm{N}^{\mathrm{O}} 3$ 
Comparing with (45) we get that

$$
\widehat{\Theta}\left(-A_{n}\right)=\Psi\left(A_{n}\right)
$$

for all $A_{n} \in S_{n}$. This proves the first part of the proposition. Furthermore, regarding $\Psi$ now as its extension to $\mathcal{S}_{n}$ and writing explicitly the equality $\widehat{\Psi}\left(D_{n}\right)=\Theta\left(D_{n}\right)$ we have for all $C_{m} \in S_{m}, D_{n} \in \mathcal{S}_{n}$

$$
\begin{array}{r}
\int_{\mathcal{S}_{n}}\left[\int_{\mathcal{S}_{m}} \widetilde{\omega}_{m}^{n, \psi}\left[\Phi, \psi ;\left(\begin{array}{ll}
A_{n} & \\
& B_{m}
\end{array}\right)\right] \psi\left[-\operatorname{Tr}\left(B_{m} C_{m}\right)\right] \mathrm{d} B_{m}\right] \\
{\left[A_{n}, C_{m}\right] \psi\left[\operatorname{Tr}\left(C_{m}^{-1} \epsilon_{m}^{n} A_{n}^{-1 t} \epsilon_{m}^{n}\right)\right] \psi\left[-\operatorname{Tr}\left(A_{n} D_{n}\right)\right] \mathrm{d} A_{n}} \\
=|2|^{\frac{1}{2} m n} \cdot \gamma(1, \bar{\psi})^{m n} \cdot \widetilde{\omega}_{n}^{m, \bar{\psi}}\left[\check{\Phi}, \bar{\psi} ;\left(w_{m} C_{m} w_{m}\right.\right. \\
\left.\left.w_{n} D_{n} w_{n}\right)\right] .
\end{array}
$$

Observing that $\left[A_{n}, C_{m}\right]=\left[A_{n}, w_{m} C_{m} w_{m}\right]$ we get the proposition by replacing $\left(C_{m}, D_{n}\right)$ with $\left(w_{m} C_{m} w_{m}, w_{n} D_{n} w_{n}\right)$.

We end this chapter with a reduction formula that we will need for the proof of the main theorem. Let $\Phi \in C_{c}^{\infty}\left(\mathcal{S}_{m+n}\right)$ and define on $S_{n} \times \mathcal{S}_{m}$ the function

$$
\Xi\left(A_{n}, B_{m}\right)=\omega_{m}^{n}\left[\Phi, \psi ;\left(\begin{array}{ll}
A_{n} & \\
& B_{m}
\end{array}\right)\right] .
$$

Associated with the action of $N_{n} \times N_{m}$ on $S_{n} \times \mathcal{S}_{m}$, we consider the generalized Kloosterman integral

$$
\omega\left[\Xi, \psi ;\left(A_{n}, B_{m}\right)\right]=\int \Xi\left({ }^{t} u_{1} A_{n} u_{1},{ }^{t} u_{2} B_{m} u_{2}\right) \theta_{n}\left(u_{1}\right) \theta_{m}\left(u_{2}\right) \mathrm{d} u_{1} \mathrm{~d} u_{2} .
$$

For a relevant element $x \in \mathcal{S}_{m+n}$ of the form $x=\left(\begin{array}{ll}x_{n} & \\ & x_{m}\end{array}\right)$, where $x_{n} \in S_{n}$ and $x_{m} \in \mathcal{S}_{m}$ are relevant, we have

$$
\omega[\Phi, \psi ; x]=\omega\left[\Xi, \psi,\left(x_{n}, x_{m}\right)\right] .
$$

\section{Proof of the main theorem}

We prove the functional equation by induction on $n$, the case $n=1$ being simply the definition of the Fourier transform. For a fixed $a_{1} \in F^{\times}$, let

$$
\begin{aligned}
\Psi\left(A_{n-1}\right)=\left[a_{1}, A_{n-1}\right] \psi\left[\operatorname{Tr}\left(a_{1}^{-1} \epsilon A_{n-1}^{-1}{ }^{t} \epsilon\right)\right] \\
\times \int \widetilde{\omega}_{1}^{n-1, \psi}\left[\Phi, \psi ;\left(\begin{array}{cc}
A_{n-1} & \\
& p_{n}
\end{array}\right)\right] \psi\left[-p_{n} a_{1}\right] \mathrm{d} p_{n}, \\
\Theta\left(D_{n-1}\right)=|2|^{\frac{1}{2}(n-1)} \cdot \gamma(1, \bar{\psi})^{n-1} \cdot \widetilde{\omega}_{n-1}^{1, \bar{\psi}}\left[\check{\Phi}, \bar{\psi} ;\left(\begin{array}{cc}
a_{1} & \\
& D_{n-1}
\end{array}\right)\right] .
\end{aligned}
$$

Applying Proposition 3.1, with $(1, n-1)$ in the role of $(m, n)$, we get that $\Psi$ extends to $\mathcal{S}_{n-1}$. In fact $\Psi, \Theta \in C_{c}^{\infty}\left(\mathcal{S}_{n-1}\right)$ and $\Theta=\check{\Psi}$. Let $a^{(1)}=$ 
$\operatorname{diag}\left(a_{2}, \ldots, a_{n}\right)$ be relevant in $\mathcal{S}_{n-1}$. By induction applied to $\Psi$ we have

$$
\begin{aligned}
& \left(K_{\psi, n-1} \widetilde{\omega}^{\psi}[\Psi, \psi ; \cdot]\right)\left(a^{(1)}\right) \\
& \quad=|2|^{\frac{1}{2}(n-1)(n-2)} \cdot \gamma(1, \bar{\psi})^{\frac{1}{2}(n-1)(n-2)} \cdot \widetilde{\omega}^{\bar{\psi}}\left[\Theta, \bar{\psi} ; a^{(1)}\right] .
\end{aligned}
$$

By (54) and (19) we have

$$
\text { (58) } \begin{aligned}
& \omega\left[\Theta, \bar{\psi} ; a^{(1)}\right]=|2|^{\frac{1}{2}(n-1)} \cdot \gamma(1, \bar{\psi})^{n-1} \cdot \gamma_{n-1}^{1}\left(-a_{1}, 1_{n-1}, \bar{\psi}\right) \cdot\left|a_{1}\right|^{\frac{1}{2}(n-1)} \\
& \times \omega\left[\omega_{n-1}^{1}[\check{\Phi}, \bar{\psi} ; \cdot], \bar{\psi} ;\left(a_{1}, a^{(1)}\right)\right] \\
&=|2|^{\frac{1}{2}(n-1)} \cdot \gamma(1, \bar{\psi})^{n-1} \cdot \gamma\left(-a_{1}, \bar{\psi}\right)^{n-1} \cdot\left|a_{1}\right|^{\frac{1}{2}(n-1)} \cdot \omega\left[\check{\Phi}, \bar{\psi} ; a_{1}, \ldots, a_{n}\right]
\end{aligned}
$$

therefore the right hand side of (57) satisfies

$$
\begin{aligned}
|2|^{\frac{1}{2}(n-1)(n-2)} \cdot \gamma(1, \bar{\psi})^{\frac{1}{2}(n-1)(n-2)} \cdot \widetilde{\omega}^{\bar{\psi}}\left[\Theta, \bar{\psi} ; a^{(1)}\right] & \\
=|2|^{\frac{1}{2} n(n-1)} \cdot & \gamma(1, \bar{\psi})^{\frac{1}{2} n(n-1)} \cdot \Gamma_{n-1}\left(-a^{(1)}, \bar{\psi}\right) \cdot\left|\sigma_{n-1}\left(a^{(1)}\right)\right|^{\frac{1}{2}} \\
& \times \gamma\left(-a_{1}, \bar{\psi}\right)^{n-1} \cdot\left|a_{1}\right|^{\frac{1}{2}(n-1)} \cdot \omega\left[\check{\Phi}, \bar{\psi} ; a_{1}, \ldots, a_{n}\right] \\
=|2|^{\frac{1}{2} n(n-1)} \cdot & \gamma(1, \bar{\psi})^{\frac{1}{2} n(n-1)} \cdot \widetilde{\omega}^{\bar{\psi}}\left[\check{\Phi}, \bar{\psi} ; a_{1}, \ldots, a_{n}\right] .
\end{aligned}
$$

Next we treat the left hand side of (57). We start with the computation of the Kloosterman integral of $\Psi$ at $x=\operatorname{diag}\left(p_{1}, \ldots, p_{n-1}\right)$. Note that

$$
\left[a_{1},{ }^{t} u x u\right] \cdot \psi\left[\operatorname{Tr}\left(a_{1}^{-1} \epsilon u^{-1} x^{-1 t} u^{-1 t} \epsilon\right)\right]=\left(\prod_{j=1}^{n-1}\left[a_{1}, p_{j}\right]\right) \psi\left[\frac{1}{a_{1} p_{n-1}}\right]
$$

for all $u \in N_{n-1}$ is constant on the orbit of $x$. So

$$
\begin{aligned}
& \widetilde{\omega}^{\psi}\left[\Psi, \psi ; p_{1}, \ldots, p_{n-1}\right]=\Gamma_{n-1}\left(-p_{1}, \ldots,-p_{n-1}, \psi\right) \\
& \times\left|\sigma_{n-1}\left(p_{1}, \ldots, p_{n-1}\right)\right|^{\frac{1}{2}}\left(\prod_{j=1}^{n-1}\left[a_{1}, p_{j}\right]\right) \psi\left[\frac{1}{a_{1} p_{n-1}}\right]
\end{aligned}
$$

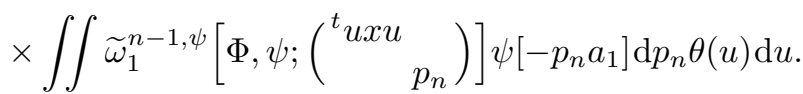

Since $\Phi$ is smooth and of compact support, it is easy to see from the right hand side of (32), that for $\phi \in C_{c}^{\infty}\left(F^{\times}\right)$the function

$$
\left(A_{n-1}, p_{n}\right) \longmapsto \phi\left(\operatorname{det} A_{n-1}\right) \cdot \widetilde{\omega}_{1}^{n-1, \psi}\left[\Phi, \psi ;\left(\begin{array}{cc}
A_{n-1} & \\
& p_{n}
\end{array}\right)\right]
$$

is smooth of compact support on $\operatorname{GL}(n-1, F) \times F$. Since the orbit ${ }^{t} u x u$, $u \in N_{n-1}$ has fixed determinant, the double integral in (60) is absolutely

TOME $133-2005-\mathrm{N}^{\mathrm{O}} 3$ 
convergent and we may switch order of integration. From (54) we get

$$
\begin{aligned}
& \Gamma_{n-1}\left(-p^{(n)}, \psi\right) \cdot\left|\sigma_{n-1}\left(p_{1}, \ldots, p_{n-1}\right)\right|^{\frac{1}{2}} \\
& \times \int \widetilde{\omega}_{1}^{n-1, \psi}\left[\Phi, \psi ;\left(\begin{array}{ll}
{ }^{t} u x u & \\
& p_{n}
\end{array}\right)\right] \theta(u) \mathrm{d} u \\
&=\Gamma_{n-1}\left(-p^{(n)}, \psi\right) \cdot\left|\sigma_{n-1}\left(p_{1}, \ldots, p_{n-1}\right)\right|^{\frac{1}{2}} \cdot \gamma_{1}^{n-1}(-x, 1, \psi) \\
& \quad \times|\operatorname{det} x|^{\frac{1}{2}} \int \omega_{1}^{n-1}\left[\Phi, \psi ;\left({ }^{t} u x u\right.\right. \\
& \\
&\left.\left.p_{n}\right)\right] \theta(u) \mathrm{d} u \\
&=\widetilde{\omega}^{\psi}\left[\Phi, \psi ; p_{1}, \ldots, p_{n}\right] .
\end{aligned}
$$

By switching order of integration in the right hand side of (60) we get that

$$
\begin{aligned}
& \widetilde{\omega}^{\psi}\left[\Psi, \psi ; p_{1}, \ldots, p_{n-1}\right] \\
& \quad=\left(\prod_{j=1}^{n-1}\left[a_{1}, p_{j}\right]\right) \psi\left[\frac{1}{a_{1} p_{n-1}}\right] \int \widetilde{\omega}^{\psi}\left[\Phi, \psi ; p_{1}, \ldots, p_{n}\right] \psi\left[-p_{n} a_{1}\right] \mathrm{d} p_{n} .
\end{aligned}
$$

Finally, we see that

(64) $\left(K_{\psi, n-1} \widetilde{\omega}^{\psi}[\Psi, \psi ; \cdot]\right)\left(a^{(1)}\right)$

$$
\begin{aligned}
& =\int\left(\prod_{j=1}^{n-1}\left[a_{1}, p_{j}\right]\right) \psi\left[\frac{1}{a_{1} p_{n-1}}\right] \int \widetilde{\omega}^{\psi}\left[\Phi, \psi ; p_{1}, \ldots, p_{n}\right] \psi\left[-p_{n} a_{1}\right] \mathrm{d} p_{n} \\
& \quad \times \psi\left[-\sum_{i=1}^{n-1} p_{i} a_{n+1-i}+\sum_{i=1}^{n-2} \frac{1}{p_{i} a_{n-i}}\right]\left(\prod_{i=2}^{n-1} \prod_{j=1}^{n-i}\left[a_{i}, p_{j}\right]\right) \mathrm{d} p_{n-1} \cdots \mathrm{d} p_{1} \\
& =\left(K_{\psi, n} \widetilde{\omega}^{\psi}[\Phi, \psi ; \cdot]\right)\left(a_{1}, \ldots, a_{n}\right) .
\end{aligned}
$$

The theorem now follows from (57), (59) and (64).

Corollary 1.3 is now immediate since $\gamma(1, \psi) \cdot \gamma(1, \bar{\psi})=1$.

\section{A formula for the smallest orbits}

Let $\kappa(n)=\frac{1}{2} n(n+1)-1$.

Proposition 5.1. - For $\Phi \in C_{c}^{\infty}\left(\mathcal{S}_{n}\right)$, the function $\phi(a)=\omega\left[\Phi, \psi, w_{n} a\right]$ is a smooth function of compact support on $F^{\times}$. Furthermore, it satisfies the functional equation

$$
|a|^{\kappa(n)} \cdot \omega\left[\Phi, \psi, w_{n} a\right]=\int \omega\left[\check{\Phi}, \bar{\psi} ;\left(\begin{array}{l}
-w_{n-1} a^{-1} \\
b
\end{array}\right)\right] \mathrm{d} b .
$$

Proof. - We can write $\phi$ as follows

$$
\omega\left[\Phi, \psi, w_{n} a\right]=\int \Phi\left(m^{\prime}\right) \psi\left(\sum_{i=2}^{n} x_{i, n+2-i}\right) \underset{i+j \geq n+2}{\otimes} \mathrm{d} x_{i, j}
$$

BULLETIN DE LA SOCIÉtÉ MATHÉMATIQUE DE FRANCE 
where

$$
m_{i, j}^{\prime}= \begin{cases}0 & \text { if } i+j \leq n, \\ a & \text { if } i+j=n+1, \\ a x_{i, j} & \text { if } i+j \geq n+2,\end{cases}
$$

and $x_{i, j}=x_{j, i}$ if $i+j \geq n+2$. Let

$$
\kappa_{1}(n)=\left\{\begin{array}{ll}
\frac{1}{4} n^{2} & \text { if } n-\text { even, } \\
\frac{1}{4}\left(n^{2}-1\right) & \text { if } n \text { - odd, }
\end{array} \quad \kappa_{2}(n)=\kappa_{1}(n-1)+n-1 .\right.
$$

Then $\kappa(n)=\kappa_{1}(n)+\kappa_{2}(n)$. After a change of variables (66) can be writen as

$$
\omega\left[\Phi, \psi, w_{n} a\right]=|a|^{-\kappa_{1}(n)} \int \Phi(m) \psi\left(a^{-1} \sum_{i=2}^{n} x_{i, n+2-i}\right) \underset{i+j \geq n+2}{\otimes} \mathrm{d} x_{i, j}
$$

where

$$
m_{i, j}=\left\{\begin{array}{cl}
0 & \text { if } i+j \leq n \\
a & \text { if } i+j=n+1, \\
x_{i, j} & \text { if } i+j \geq n+2,
\end{array}\right.
$$

and $x_{i, j}=x_{j, i}$ if $i+j \geq n+2$. The smoothness of $\phi$ follows from the fact that $\Phi$ is smooth. Also $\Phi(m)=0$ for large enough $|a|$ and for all $x_{i, j}$ as above, since $\Phi$ is of compact support. Therefore $\phi(a)$ vanishes when $|a|$ is sufficiently large. Let $X$ be the $n \times n$ symmetric matrix with 0 in the $(i, j)$-th entry whenever $i+j \leq n+1$ and $x_{i, j}$ in the $(i, j)$-th entry whenever $i+j \geq n+2$. Let $Z$ be a similar variable matrix with entries $z_{i, j}$, thus

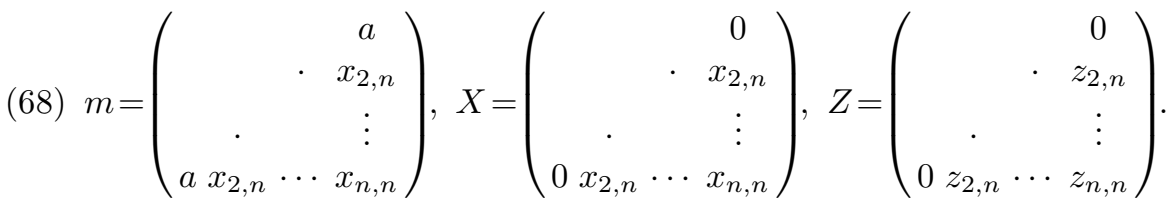

Since $\Phi$ is smooth and of compact support, there are integers $\ell<k$ such that $\Phi(m)=0$ unless $x_{i, j} \in \wp^{\ell}$ and $\Phi(m+Z)=\Phi(m)$ whenever $z_{i, j} \in \wp^{k}$ for all $i, j$. Therefore,

$$
\phi(a)=\sum_{x_{i, j} \in \wp^{\ell} / \wp^{k}} \Phi(m) \int_{z_{i, j} \in \wp^{k}} \psi\left(a^{-1} \sum_{i=2}^{n}\left(x_{i, n+2-i}+z_{i, n+2-i}\right)\right) \underset{i+j \geq n+2}{\otimes} \mathrm{d} z_{i, j} .
$$

This integral factors, for example, through the integral $\int_{\wp^{k}} \psi\left(a^{-1} z_{2, n}\right) \mathrm{d} z_{2, n}$ which vanishes whenever $|a|$ is small enough. So we get also that $\phi(a)=0$ for $|a|$ sufficiently small. We can write

$$
\int \omega\left[\check{\Phi}, \bar{\psi} ;\left(\begin{array}{l}
-w_{n-1} a^{-1} \\
b
\end{array}\right)\right] \mathrm{d} b=\int \breve{\Phi}\left(p^{\prime}\right) \psi\left(-\sum_{i=1}^{n} y_{i, n+1-i}\right) \underset{2 n>i+j \geq n+1}{\otimes} \mathrm{d} y_{i, j} \mathrm{~d} b
$$

TOME $133-2005-\mathrm{N}^{\mathrm{O}} 3$ 
where

$$
p_{i, j}^{\prime}= \begin{cases}0 & \text { if } i+j \leq n-1 \\ -a^{-1} & \text { if } i+j=n \\ -a^{-1} y_{i, j} & \text { if } 2 n>i+j \geq n+1 \\ b & \text { if } i+j=2 n\end{cases}
$$

and $y_{i, j}=y_{j, i}$ if $i+j \geq n+1$. After a change of variables this becomes

$\int \omega\left[\check{\Phi}, \bar{\psi} ;\left(\begin{array}{rr}-w_{n-1} a^{-1} & \\ & b\end{array}\right)\right] \mathrm{d} b=|a|^{\kappa_{2}(n)} \int \check{\Phi}\left(p^{\prime \prime}\right) \psi\left(a \sum_{i=1}^{n} y_{i, n+1-i}\right) \underset{i+j \geq n+1}{\otimes} \mathrm{d} y_{i, j}$

where

$$
p_{i, j}^{\prime \prime}= \begin{cases}0 & \text { if } i+j \leq n-1, \\ -a^{-1} & \text { if } i+j=n, \\ y_{i, j} & \text { if } i+j \geq n+1,\end{cases}
$$

and $y_{i, j}=y_{j, i}$ if $i+j \geq n+1$ which is the same as writing

$$
\begin{aligned}
\int \omega\left[\check{\Phi}, \bar{\psi} ;\left(\begin{array}{rr}
-w_{n-1} a^{-1} \\
b
\end{array}\right)\right] \mathrm{d} b \\
\quad=|a|^{\kappa_{2}(n)} \int \hat{\Phi}(p) \psi\left(a \sum_{i=1}^{n} y_{i, n+1-i}\right) \underset{i+j \leq n+1}{\otimes} \mathrm{d} y_{i, j}
\end{aligned}
$$

where

$$
p_{i, j}= \begin{cases}y_{i, j} & \text { if } i+j \leq n+1 \\ -a^{-1} & \text { if } i+j=n+2, \\ 0 & \text { if } i+j \geq n+3\end{cases}
$$

and $y_{i, j}=y_{j, i}$ if $i+j \leq n+1$. Next let $Y$ be the $n \times n$ symmetric matrix with

$$
Y_{i, j}= \begin{cases}y_{i, j} & \text { if } i+j \leq n+1 \\ 0 & \text { if } i+j \geq n+2\end{cases}
$$

Let $A^{\prime}$ be defined by $p=Y+A^{\prime}$ and let $X$ and $Z$ be matrix variables as defined in (68). Thus

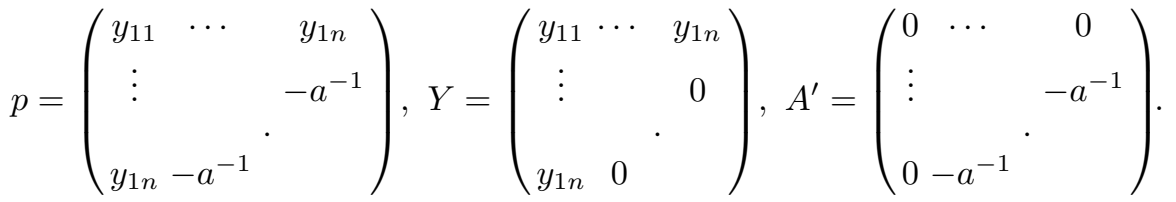

BULLETIN DE LA SOCIÉTÉ MATHÉMATIQUE DE FRANCE 
Using Fourier inversion formula for the function $\hat{\Phi}(Y+(\cdot))$ in the $(i, j)$-th entries for all $i+j \geq n+2$, we obtain from (69) that

$$
\begin{aligned}
& |a|^{-\kappa_{2}(n)} \int \omega\left[\check{\Phi}, \bar{\psi} ;\left(\begin{array}{rr}
-w_{n-1} a^{-1} & \\
\end{array}\right)\right] \mathrm{d} b \\
& =\int \widehat{\Phi}(Y+Z) \psi[-\operatorname{Tr}(X Z)] \mathrm{d} Z \psi\left[\operatorname{Tr}\left(X A^{\prime}\right)\right] \mathrm{d} X \psi\left(a \sum_{i=1}^{n} y_{i, n+1-i}\right) \underset{i+j \leq n+1}{\otimes} \mathrm{d} y_{i, j} .
\end{aligned}
$$

Let $A=w_{n} a$, one easily observes now that $\operatorname{Tr}(Z A)=\operatorname{Tr}(Y X)=0$ and therefore $\operatorname{Tr}((Y+Z)(A-X))=\operatorname{Tr}(Y A-Z X)=a \sum_{i=1}^{n} y_{i, n+1-i}-\operatorname{Tr}(X Z)$, so we get by the Fourier inversion formula applied to $\hat{\Phi}$ that

$$
\begin{aligned}
& |a|^{-\kappa_{2}(n)} \int \omega\left[\check{\Phi}, \bar{\psi} ;\left(\begin{array}{rr}
-w_{n-1} a^{-1} & \\
b
\end{array}\right)\right] \mathrm{d} b \\
& =\int \widehat{\Phi}(Y+Z) \psi[\operatorname{Tr}((Y+Z)(A-X))] \mathrm{d} Y \mathrm{~d} Z \psi\left[\operatorname{Tr}\left(X A^{\prime}\right)\right] \mathrm{d} X \\
& =\int \Phi(A-X) \psi\left[\operatorname{Tr}\left(X A^{\prime}\right)\right] \mathrm{d} X=\int \Phi(A+X) \psi\left[\operatorname{Tr}\left(-X A^{\prime}\right)\right] \mathrm{d} X .
\end{aligned}
$$

But $m=A+X$ and $\operatorname{Tr}\left(-X A^{\prime}\right)=a^{-1} \sum_{i=2}^{n} x_{i, n+2-i}$, so comparing with (67), the right hand side of $(70)$ is equal to $|a|^{\kappa_{1}(n)} \omega\left[\Phi, \psi ; w_{n} a\right]$.

\section{BIBLIOGRAPHY}

[1] Flicker (Y.Z.) \& Kazhdan (D.A.) - Metaplectic correspondence, Publ. Math. Inst. Hautes Études Sci., t. 64 (1986), pp. 53-110.

[2] JACQUet (H.) - Facteurs de transfert pour les intégrales de Kloosterman, C. R. Acad. Sci. Paris Sér. I Math., t. 336 (2003), no. 2, pp. 121-124.

[3] _ Smooth transfer of Kloosterman integrals, Duke Math. J., t. 120 (2003), no. 1, pp. 121-152.

[4] Kloosterman identities over a quadratic extension, Ann. Math. (2), t. 160 (2004), no. 2, pp. 755-779.

[5] Kazhdan (D.A.) \& Patterson (S.J.) - Metaplectic forms, Publ. Math. Inst. Hautes Études Sci., t. 59 (1982), pp. 35-142.

[6] Mao (Z.) - A fundamental lemma for metaplectic correspondence, J. reine angew. Math., t. 496 (1998), pp. 107-129.

[7] WeIL (A.) - Sur certains groupes d'opérateurs unitaires, Acta Math., t. 111 (1964), pp. 143-211. 